\title{
Transcript Profiles of Auxin Efflux Carrier and IAA-Amido Synthetase Genes Suggest the Role of Auxin on Apple (Malus $\times$ domestica) Fruit Maturation Patterns
}

\author{
Sungbong Shin1, Jinwook Lee ${ }^{1,2}$, Dave Rudell1, Kate Evans ${ }^{3}$, Yanmin Zhu1* \\ ${ }^{1}$ Tree Fruit Research Laboratory, United States Department of Agriculture, Wenatchee, WA, USA \\ ${ }^{2}$ Department of Horticulture Science, Kyungpook National University, Daegu, Republic of Korea \\ ${ }^{3}$ Tree Fruit Research and Extension Center, Washington State University, Wenatchee, WA, USA

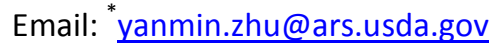

Received 21 November 2014; accepted 10 March 2015; published 17 March 2015

Copyright (C) 2015 by authors and Scientific Research Publishing Inc.

This work is licensed under the Creative Commons Attribution International License (CC BY). http://creativecommons.org/licenses/by/4.0/

\section{(c) (i) Open Access}

\begin{abstract}
Auxin has been suggested to play an essential role in regulating apple fruit maturation and ripening, though the molecular function of auxin and its interaction with ethylene during apple fruit development are largely unknown. To understand the function of auxin during apple fruit maturation and ripening, auxin efflux carrier and IAA-amido synthetase encoding genes were identified from the apple genome based on the results of previous microarray analysis. The expression patterns of these genes were analyzed using QRT-PCR during 10 - 12 weeks of fruit maturation for two apple cultivars: "Golden Delicious" (GD) and "Cripps Pink" (CP), which have the distinct patterns of maturation progression. Our results showed that the expressions of auxin efflux carrier and IAA-amido synthetase genes have a correlation with the timing of ethylene biosynthesis pathway activation in both cultivars. The earlier and stronger expression of MdGH3.102 and MdAECFP1 in the fruit of GD, a mid-season cultivar, correlates with the earlier activation of a pre-climacteric ethylene biosynthesis gene of MdACS3, compared with that in $\mathrm{CP}$, a late-ripening apple cultivar. Results of exogenous IAA treatment indicated that the expression patterns of the genes were regulated in a fruit maturity dependent manner. Our results suggested that the dynamics of the auxin level in apple fruit cortex could be one of the key factors influencing the timing of ethylene biosynthesis pathway activation and consequently contributed to the control of the apple maturation progression.
\end{abstract}

\footnotetext{
${ }^{*}$ Corresponding author.
} 


\section{Keywords}

\section{Auxin Transport, Auxin-Amino Acid Conjugation, Ethylene Biosynthesis, Fruit Maturation, Quantitative Gene Expression}

\section{Introduction}

Fruit maturation and ripening are tightly regulated by multiple genetic factors [1]-[3]. Under specific cellular contexts, such as in maturing apple fruit, the modulation of the gene expression depends on the integration of multiple inputs of developmental or environmental signals. Among these signals, crosstalk between plant hormones is believed to be one of the important aspects which control fruit development [4]-[7]. Specific to climacteric fruit such as apple, ethylene is well-known to play a pivotal role in the ripening regulation [8]-[11]. A previous study on the expression patterns of a pre-climacteric ethylene biosynthesis gene, i.e. aminocyclopropane-1-carboxylic acid synthase gene (MdACS3) among 12 apple cultivars suggested that MdACS3 acts as a maturation "accelerator" by triggering the expression of MdACS1 [12]. However, ethylene itself is most likely just one node in the hormone regulation network. Recent transcriptomic and genomic studies indicated the roles of auxin and its potential interactions with ethylene during apple fruit maturation and ripening [13] [14], but the details of the interactions are unknown.

Auxin is a prominent plant hormone that regulates various aspects of plant growth and development by transmitting intrinsic and environmental signals into physiological processes [15]-[19]. Auxin action in plants depends on the cellular auxin level which is determined by the coordination of auxin transport and metabolism [17]. Auxin transporters create unique asymmetric gradients in plant tissues which result in directional distribution [20]-[24]. PIN (pin-formed), a well-studied auxin transporter protein, plays a central role in auxin transport [23], and the differential expression of the PIN gene family as well as the polar and subcellular distribution of PIN proteins is important aspects of the regulation [25]-[27]. The role of PIN as an IAA (indole-3-acetic acid) transporter has been well characterized in various plant species including Arabidopsis [20], rice [21], and maize [22]. In addition, the cellular auxin level can be regulated by auxin metabolism and homeostasis including biosynthesis, conjugation and degradation [16] [17] [28]. For example, the conjugation of amino acids to free IAA by the function of GH3 (Gretchen Hagen 3, IAA-amido synthetase) proteins controls the availability of biologically active auxin in the cell at specific times [29].

It has been suggested that auxin might play an important role in fruit development and ripening [30]-[32]. The role of auxin in cell division and cell enlargement during fruit development has been well elucidated with auxin treatment studies [13] [33]. Specific distribution of auxin in fruit tissues by the function of auxin transporters has been shown to regulate fruit development and ripening [34]-[39]. However, crosstalk among hormones rather than individual action is most likely to be the common theme regulating most biological processes including fruit ripening [31] [32] [40]-[42]. In particular, auxin-ethylene interaction in plants has been well-documented [43]-[45], though very little is known in maturing apple fruit. For example, the auxin-responsive behavior of ACS (1-aminocyclopropane-1-carboxylate synthase) genes in melon and peach was shown with auxin treatment [46] [47]. Specific to apple fruit maturation and ripening process, each apple cultivar produces ethylene at the end of maturation and the onset of ripening, but the timing of ethylene production can vary by up to three months even among the elite commercial cultivars. It is conceivable that the crosstalk between auxin and ethylene in maturing apple fruit is critical for cultivar-specific ripening dynamics. The hypothesis tested in this study is that the regulation of the auxin availability by the expression patterns for auxin efflux carrier and IAA-amido synthetase genes contributes to the control of the timing of ethylene biosynthesis pathway activation in apple fruit tissues, and therefore the cultivar-specific apple fruit ripening season.

In the current study, based on our previous transcriptome analysis of apple fruit ripening [14], the gene families of auxin efflux carrier and IAA-amido synthetase were identified from the apple genome sequences [48]. The expression profiles of apple auxin efflux carriers (MdPIN1 and MdAECFP1) and IAA-amido synthetases (MdGH3.102 and MdGH3.18), as well as ethylene biosynthesis genes (MdACS3 and MdACS1), were characterized using the qRT-PCR (quantitative reverse transcription polymerase chain reaction). The expression patterns of these genes were compared between two distinct apple cultivars (“Golden Delicious" and "Cripps Pink") 
from 86 to 183 days after full bloom (DAFB). The effect of exogenous IAA treatment added to our understanding on the expression regulation of these genes during apple fruit maturation.

\section{Materials and Methods}

\subsection{Fruit Maturity Data and Tissue Collection}

Fruit of two different apple (Malus $\times$ domestica Borkh.) cultivars, "Golden Delicious" (GD) and "Cripps Pink" (CP), with uniform size and appearance were sampled from two commercial orchards near Quincy, WA during the 2011 growing season. Based on their projected ripening times, weekly fruit samples were collected between 86 - 162 DAFB (days after full bloom) for GD and between 99 - 183 DAFB for CP. Each weekly sample included fifteen apples. Fruit maturity stages were evaluated using starch pattern index (SPI), internal ethylene concentration (IEC), fruit diameter and fruit firmness. SPI is a 1 - 6 scale where " 1 " represents no starch degradation and " 6 " indicates complete starch degradation [49]. Fruit firmness and diameter were assessed with a Mohr ${ }^{\circledR}$ Digi-Test instrument (Mohr and Associates, Richland, WA). IEC was determined by withdrawing a 0.5 $\mathrm{mL}$ gas sample from the core cavity of each fruit which was then injected into an Agilent 6890A Series gas chromatograph (Agilent Technologies, Inc., Wilmington, DE). The glass GC column was $0.3 \mathrm{~cm} \times 30 \mathrm{~cm}$ i.d., packed with 80 - 100 mesh Porapak q. Flow rates for $\mathrm{N}_{2}$ (carrier) and $\mathrm{H}_{2}$ gases were $30 \mathrm{~mL} / \mathrm{min}$, and for air 300 $\mathrm{ml} / \mathrm{min}$ [50]. Fruit cortex tissue was collected as most maturity data is based on the maturity changes in cortex. Collected tissues were immediately frozen in liquid nitrogen, and afterwards stored at $-80^{\circ} \mathrm{C}$ until RNA isolation.

\subsection{IAA Treatment}

For exogenous IAA (indole-3-acetic acid) treatment, fruit of both GD and CP were sampled at DAFB 127 days. The fruit maturity level for collected samples including IEC and SPI was measured as mentioned above. IAA treatment was performed by injecting $1 \mathrm{ml}$ of $0.1 \mu \mathrm{M}$ IAA (Sigma-Aldrich, St. Louis, MO) in $0.1 \%$ ethanol into apple fruit through the calyx using a $1 \mathrm{ml}$ syringe. The control group was injected with $0.1 \%$ ethanol without IAA. Both control and IAA-injected apples were held at room temperature for 0, 1, 3, 7, and 14 days. Cortex tissue from the apples was separated, immediately frozen in liquid nitrogen, and afterwards stored at $-80^{\circ} \mathrm{C}$ until RNA isolation.

\subsection{Identifying Candidate Genes from the Apple Genome and Sequence Analysis}

Based on the previous apple transcriptome analysis on apple fruit ripening [14], unigene and EST sequences with annotated function of auxin conjugation and auxin transporter were identified from the apple genome sequences by performing a BLASTN search against Apple Genome V 1.0 predicted CDS sequences in the Genome Database for Rosaceae website (www.rosaceae.org) [48]. Name of the genes were followed [13] [37]. Based on the corresponding amino acid sequence for each gene, protein domain structure was predicted with SMART and Pfam, and the potential subcellular location was predicted with PSORT and TargetP (www.expasy.org).

\subsection{Gene-Specific Primer Design}

Forward and reverse primers for MdACS1, MdACS3, MdPIN1, MdAECFP1, MdGH3.18, MdGH3.102 and MdActin (Table 1) were designed using web-based software Primer3plus (www.bioinformatics.nl/cgi-bin/primer3plus/primer3plus.cgi) and the IDT oligo analyzer (www.idtdna.com/analyzer/Applications/OligoAnalyzer/). Where possible, an optimum annealing temperature of $60^{\circ} \mathrm{C}$, GC content $40 \%$ - 60\%, amplicon length 150 - $180 \mathrm{bp}$, and primer length 20 bp were applied. DNA sequences for genes in each family were aligned by ClustalW (www.ebi.ac.uk/clustalw) and BLAST (www.ncbi.nlm.nih.gov/BLAST/) to select the divergent regions at or close to the 3' UTR (un-translated region) for the gene-specific primer design. Amplicons were sequenced to confirm the identity of the specific genes.

\subsection{RNA Isolation and Real-Time qRT-PCR}

The frozen fruit cortex tissues were ground to a fine powder in liquid nitrogen and the total RNA was isolated 
Table 1. Selected genes and gene specific primers.

\begin{tabular}{|c|c|c|c|}
\hline Gene name & $\begin{array}{c}\text { Gene bank accession or } \\
\text { EST }\end{array}$ & Gene model from apple genome & Primer sequences \\
\hline \multirow[t]{2}{*}{ MdACS1 } & L31347 & MDC019670.430 & Forward: TCACCTCAATATATCTCCTGGA \\
\hline & \multirow{3}{*}{ U73816.1 } & \multirow{3}{*}{ MDP0000145123 } & Reverse: CCTCAGGGACGTTGTAATAC \\
\hline \multirow{2}{*}{ MdACS3 } & & & Forward: TTTCGAGTTGGCACTGTG \\
\hline & & & Reverse: TCATATCTCGTCCTCAGTCTC \\
\hline \multirow{2}{*}{ MdPIN1 } & \multirow[b]{2}{*}{$\begin{array}{c}\text { Contig7586, Contig18662, } \\
\text { CV880396 }\end{array}$} & \multirow{2}{*}{ MDP0000138035 } & Forward: TGCCAGCAATTGTAGCAA \\
\hline & & & Reverse: CCTGTACAATGGCAACAT \\
\hline \multirow{2}{*}{ MdAECFP1 } & \multirow{2}{*}{ Contig17221 } & \multirow{2}{*}{ MDP0000344085 } & Forward: GTGCACTCTGACCCATTA \\
\hline & & & Reverse: CAGTGACGCGAAAGCATA \\
\hline \multirow{2}{*}{$M d G H 3.18$} & \multirow{2}{*}{ Contig3460 } & \multirow{2}{*}{ MDP0000811081 } & Forward: ACCAGTACCATTGTCACAA \\
\hline & & & Reverse: TTTCTCCTGCATATAAAGTTCAAT \\
\hline \multirow{2}{*}{ MdGH3.102 } & \multirow{2}{*}{ CN909148 } & \multirow{2}{*}{ MDP0000872868 } & Forward: CCAAGGTGTGTGAATTTCT \\
\hline & & & Reverse: GCTATAGCCACCACAACA \\
\hline \multirow{2}{*}{ MdActin } & \multirow{2}{*}{ CV882335 } & \multirow{2}{*}{ MDP0000752428 } & Forward: GTCGTACTACTGGTATCGTT \\
\hline & & & Reverse: TCATAGTCAAGAGCAATGTA \\
\hline
\end{tabular}

following the modified method [51]. Total RNA was quantified using a ND 1000 Nanodrop spectrophotometer (NanoDrop Technologies, Wilmington, DE) and RNA quality was verified by agarose gel electrophoresis. Total RNA was treated with DNase I (Qiagen, Valencia, CA) and then purified with RNeasy cleanup columns (Qiagen, Valencia, CA). Two $\mu \mathrm{g}$ of DNase-treated RNA was used to synthesize first-strand cDNA using SuperScript $^{\mathrm{TM}}$ II reverse transcriptase (Invitrogen, Grand Island, NY) and poly dT (Operon, Huntsville, AL) as the primer. The cDNA was diluted 20 times and a $0.6 \mu \mathrm{L}$ aliquot was used in a $15 \mu \mathrm{L}$ quantitative PCR (qPCR) reaction mix: $0.45 \mu \mathrm{L}$ SYBR Green I dye (Invitrogen, Grand Island, NY), 1× iTaq buffer (Biorad, Hercules, CA), $0.2 \mathrm{mM}$ dNTP (Applied Biosystem, Waltham, MA), $2.5 \mathrm{mM} \mathrm{MgCl}_{2}, 0.3$ units of iTaq DNA polymerase (Biorad, Hercules, CA) and $0.2 \mu \mathrm{M}$ forward/reverse primer (IDT, Coralville, IA). Quantitative reverse transcription PCR (qRT-PCR) amplification and detection was performed as described [12]. Dissociation curves were run for each primer set to verify the specific amplification. Each sample was represented by two independent total RNA isolations converted into two separate cDNAs. Each cDNA sample set was amplified in triplicate in a 96 well reaction plate and the PCR reaction was repeated twice for multi-plate analysis. Target gene expression was normalized to that of the internal reference gene (MdActin) using the $2^{-\Delta \Delta C T}$ method. The comparable normalized Ct values (within the data series for either cultivar) were selected as "adaptive” or "flexible” calibrator for better comparability between cultivars, in terms of the relative gene expression level. As a result of using "adaptive" calibrators and the same reference gene, the change of the transcript abundance for the studied genes (expressed in fold change) were more comparable between the two cultivars.

\subsection{Statistical Analysis}

Fruit maturity data was analyzed using SAS version 9.2 (SAS Institute, Cary, NC, USA). All data was subjected to analysis of variance (ANOVA) in order to determine the main and interaction effects of cultivar/fruit developmental stages. Duncan's multiple range tests were applied to determine the comparison of means at a significance level of 0.05. Responses of IEC, fruit firmness, SPI and fruit diameter to DAFB were modeled using linear and quadratic regression analyses.

\section{Results and Discussion}

\subsection{Physiological Characterization of Fruit Maturation Progression for GD and CP}

Based on the maturity data during ten-week apple fruit maturation and ripening, two apple cultivars, i.e. “Gol- 
den Delicious” (GD) and “Cripps Pink” (CP), demonstrated different progression of fruit development. Although both cultivars had full bloom in the first week of May in 2011, the fruit ripening time differed by almost a month, i.e. early Oct for GD and early Nov for CP. Fruit IEC (internal ethylene concentration) for both cultivars showed a low-level fluctuation which is typical in pre-climacteric apple fruit. Fruit firmness is characteristically decreasing along the maturity progression though their actual values between two cultivars were with a substantial difference (Figure 1 and Table 2). The SPI (starch pattern index) values, a common fruit maturation indicator, demonstrated a significant difference between two cultivars: a steady increase of SPI value since 111 DAFB (days after full bloom) for GD and the late-initiated changes of SPI values after 147 DAFB in CP. Fruit diameter also exhibited steady increase but with significant differences between the two cultivars.

Several physiological indicators have been used to define apple fruit maturity in practice, such as DAFB, fruit firmness, background color, IEC, and SPI. Fruit firmness is specific to a cultivar but generally decreases along
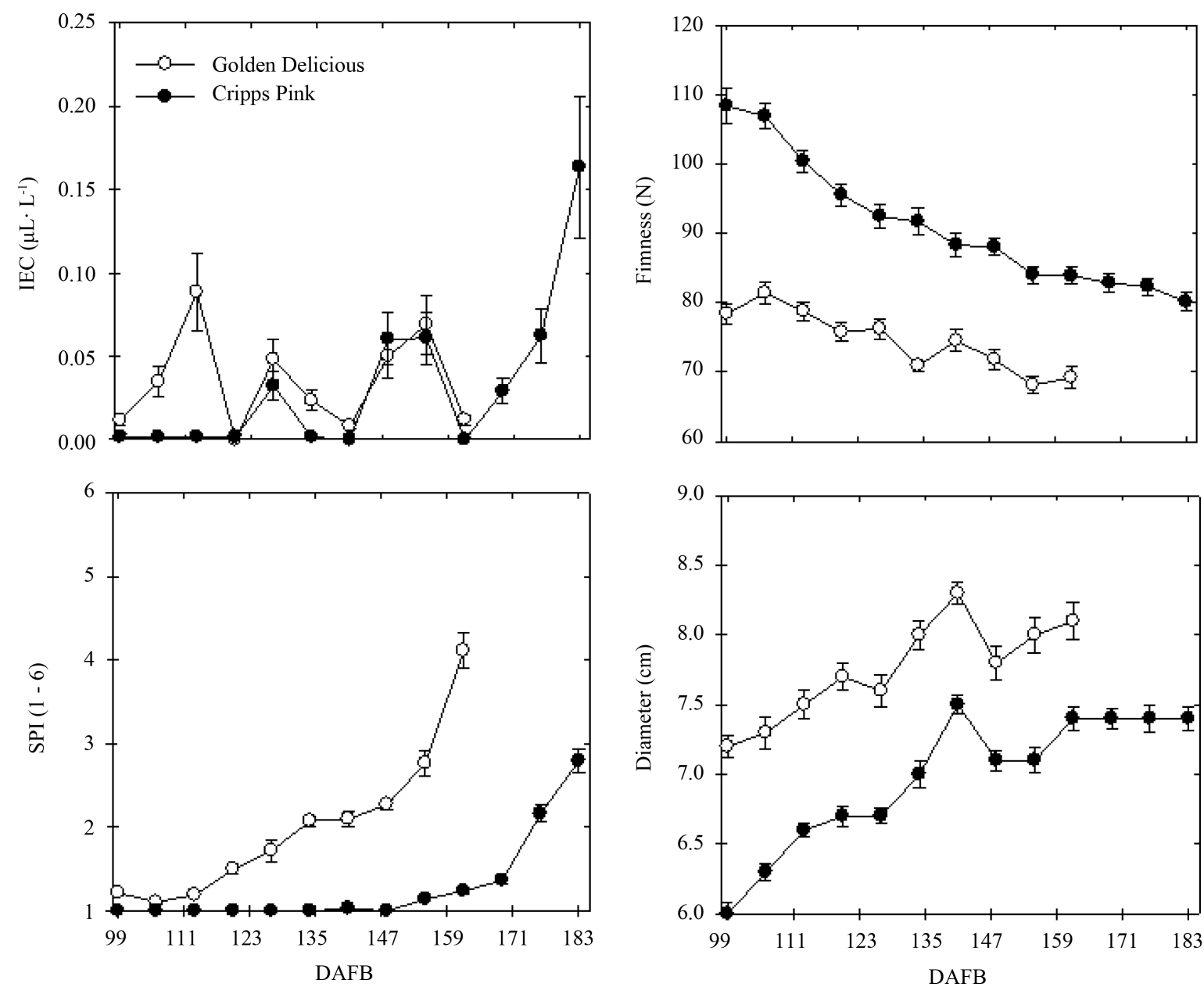

Figure 1. Fruit maturity of "Golden Delicious" (GD) and "Cripps Pink" (CP) was characterized in terms of internal ethylene concentration (IEC), fruit firmness (N), starch pattern index (SPI, based on 1 - 6 scale) and fruit diameter (cm). DAFB: days after full bloom. Each data point represents the mean of 15 fruits $(n=15)$ with standard error. The empty circle represents "Golden Delicious”; the black circle represents “Cripps Pink”.

Table 2. Fruit maturity of “Golden Delicious” and “Cripps Pink” apple cultivars.

\begin{tabular}{ccccc}
\hline Factors & IEC & Fruit firmness & SPI & Fruit diameter \\
\hline Cultivar & NS & $*^{* * *}$ & ${ }^{* * * *}$ & $* * *$ \\
Week & $* * * * * * * * *$ & $* * * * *$ \\
\hline
\end{tabular}

IEC: stand for internal ethylene concentration; SPI: starch pattern index; NS, non-significant; ${ }^{* * * *}$ significant at $P<0.0001$. 
the maturation process. IEC remains a low and fluctuating level before physiological maturity [12] [52]. Steadyprogressed SPI [59] is arguably the more objective evaluation of fruit maturity which is commonly used as an industry standard for determining commercial harvest time. In this study, the change of SPI for both cultivars showed substantial variations during the maturation, with the earlier and more progressive change in GD and a slow and delayed change in CP. Fruit quality such as at-harvest fruit firmness and postharvest storability are generally associated with fruit maturation patterns and harvest seasons [53]. The ripening time of apple fruit is a simple but important phenotype with practical and economic implications, but the genetic controls and molecular regulations behind this trait are still largely elusive.

\subsection{Identified Auxin Efflux Carrier and IAA-Amido Synthetase Genes from the Apple Genome Sequences}

The full-length sequences of auxin efflux carrier and IAA-amido synthetase gene family members were identified from the apple draft genome sequences [48]. MdPIN1 (MDP0000138035) was identified from apple genome sequences based on the sequence similarity with two contigs and one singlet identified from the previous microarray anlysis [14]. Similarly, the full-length sequences of a MdAECFP1 (auxin efflux carries family protein, MDP0000344085) and two genes encoding enzymes functioning in auxin and amino acid conjugation [MdGH3.102 (MDP0000872868) and MdGH3.18 (MDP0000811081)] were identified based on the identified EST sequences [14]. For tracking the activation of the ethylene biosynthesis pathway and potential correlation between auxin and ethylene function during apple fruit maturation, two ethylene biosynthesis genes, i.e. MdACS3 and MdACS1, were included in the experiment. The genomic information of these genes and primer sequences were shown in Table 1; the sequence features including their putative sub-cellular location, functional domain, and homologous genes from other plant species were shown on Table 3.

The gene-specific primers, that were designed based on the multi-sequence alignment of all gene family members, have been shown to generate unambiguous PCR product for reliable evaluation of gene expression levels. The sequences of the amplified regions seem to be identical between these two cultivars using qRT-PCR method. The internal reference of an apple MdActin (MDP0000752428) has been used in several previous studies and its consistency among various tissue types and treatments has been tested in several studies [54]-[56]. Additionally, use of "adaptive calibrator" for calculating the relative expression values enhances the comparability of the relative gene expression levels between two cultivars.

\subsection{The Expression of Auxin-Related Genes and Ethylene Biosynthesis Genes during Apple Fruit Maturation}

The expression patterns of auxin efflux carrier (MdPIN1 and MdAECFP1) and IAA-amido synthetase genes (MdGH3.102 and MdGH3.18), as well as ethylene biosynthesis genes (MdACS1 and MdACS3), were profiled during apple maturation (Figure 2). The expression of MdACS3, which is responsible for pre-climacteric ethylene biosynthesis, was induced at 130 DAFB in GD; there was a 4 - 5 weeks delay for the expression of MdACS3 in the fruit of CP. The expression of the climacteric ethylene biosynthesis gene, MdACS1, was highly induced around physiological maturity at SPI 3.5 or at 150 DAFB for GD, while the activation of MdACS1 was at least 4 weeks later in $\mathrm{CP}$ or in the last collected sample (Figure 2). The expression profiles of MdAECFP1 showed a steady up-regulated pattern as fruit maturity increased in both cultivars, though a substantially higher level was observed in most of the samples from the mid-season cultivar GD than in those of the late-season cultivar CP since 110 DAFB. The expression patterns of MdPIN1 exhibited a low-level induction along the entire

Table 3. Bioinformatics of auxin-related genes.

\begin{tabular}{cccc}
\hline Gene name & Sub-cellular location & Functional domains & Homologous genes \\
\hline MdPIN1 & PM & Transmembrane & AtPIN1 (At1g73590) \\
MdAECFP1 & Endoplasmic reticulum (ER) & Transmembrane & AtAECFP1 (At1g76530) \\
MdGH3.18 & Cyto/others (Chloro, Mito, Nucleus) & GH3 (Indole-3-acetic acid-amido synthetase) & OsGH3.5 (Os5g0586200) \\
MdGH3.102 & Cyto/others (Chloro, Mito, Nucleus) & GH3 (Indole-3-acetic acid-amido synthetase) & AtGH3.1 (At2g14960) \\
\hline
\end{tabular}

At: Arabidopsis thaliana; Os: Oryza sativa; PM: plasma membrane; Cyto: cytoplasm; Chloro: chloroplast; Mito: mitochondria. 

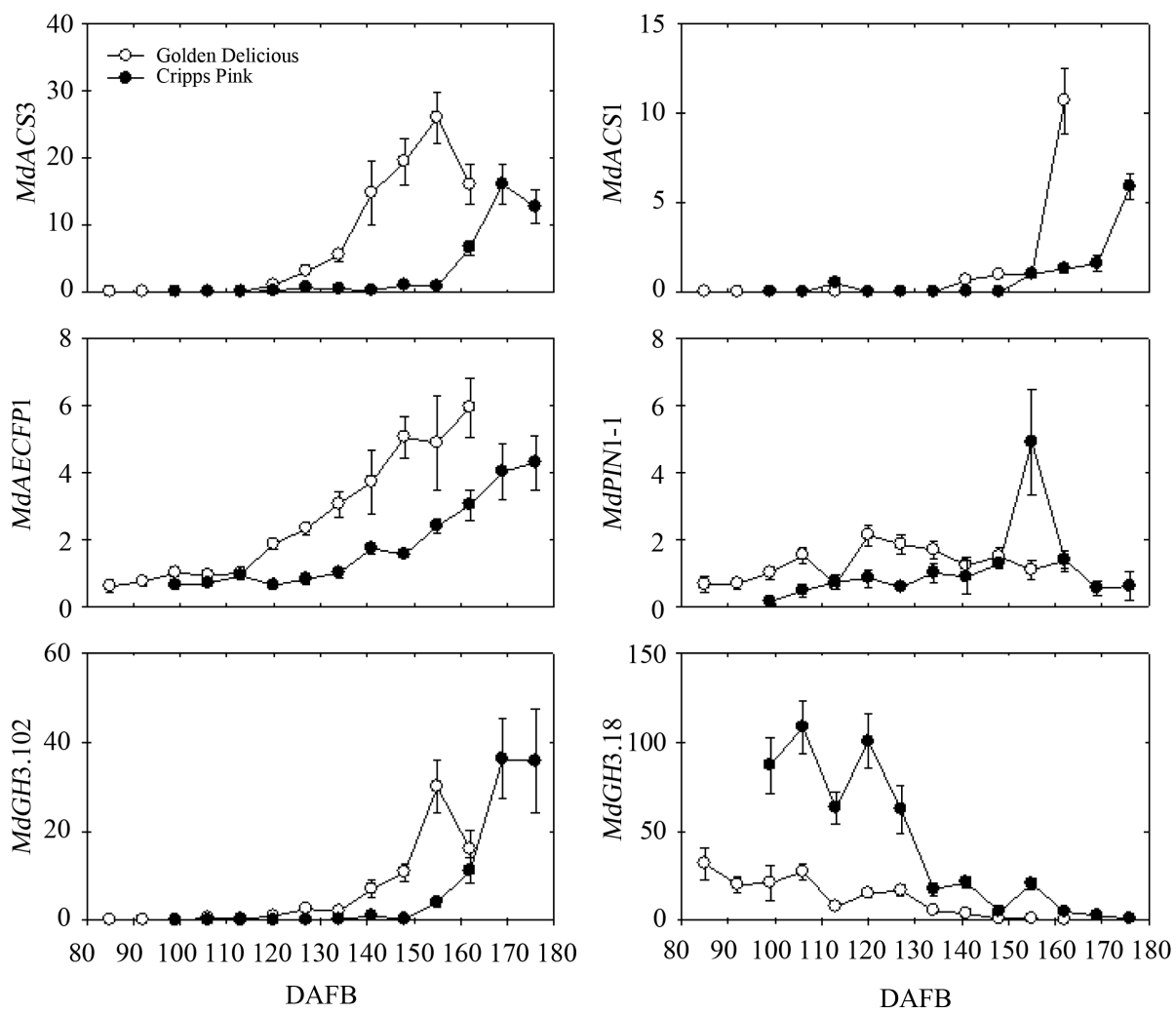

Figure 2. Gene expression profiles of two ethylene biosynthesis genes (MdACS1 and MdACS3) and four auxin efflux carrier and IAA-amido synthetase genes (MdPIN1, MdAECFP1, MdGH3.18, and MdGH3.102) in "Golden Delicious" (GD) and "Cripps Pink" (CP) apple fruit flesh (cortex) during fruit maturation. Numbers on Y axis indicate the fold change of the relative gene expression level; numbers on X axis indicate DAFB (days after full bloom). The empty circle represents GD; the black circle represents CP. Each data point represents the means of 4 replicates with standard error.

fruit maturation process, and a slightly higher expression level was observed in the fruit tissues of GD than of CP except in one sample. MdGH3.102 demonstrated increasingly up-regulated expression patterns but only at the later stages of fruit maturation after 120 DAFB for GD and 150 DAFB for CP. MdGH3.18 was highly expressed at the early stages but decreasing trend as fruit reached physiological maturity, which is opposite to the expression profile of MdGH3.18; and a much higher expression level was observed in CP than in GD.

Auxin has long been considered a natural inhibitor for fruit ripening as the concentration of auxin is low during pear ripening [57]. It was also observed that as apple ripening began, auxin concentration decreased and ethylene concentration increased [58]. Therefore, the dynamics of auxin availability and efficient removal, as well as the balance with other hormones such as jasmonate [55], could all contribute to the genotype-specific apple fruit maturation pattern or ripening season. The observations from current study indicated the more active transcriptional regulation of auxin metabolism in the mid-season cultivars GD compared with the late ripening cultivar CP. For example, the earlier and higher expression level of MdAECFP1 (functioning in putting away cellular-free auxin) and stronger up-regulation of MdGH3.102 (deactivates free auxin by making a conjugation with an amino group) in GD is correlated with the early MdACS3 activation in GD. On the other hand, possible less efficient activity of removing cellular auxin to organelles due to the weaker MdAECFP1 gene expression in CP may contribute to the delayed MdACS3 induction and late-ripening features. These observations are consistent with the hypothesis that a reduced level of biologically active auxin is the signal for ethylene biosynthesis pathway activation.

The contrasting expression profile of two auxin efflux carriers (MdAECFP1 and MdPIN1) during apple fruit maturation suggests that they have a distinct role, and sequence analysis suggests their different subcellular locations. Recent studies introduced a new auxin transporter family, group called PIN-LIKES (PILS) and distin- 
guished PILS from other PIN families based on their central hydrophilic loop length: long loop-PIN, and short loop-PILS [23] [59] [60]. Unlike PIN which are localized on the plasma membrane and might be involved in intercellular auxin transport to form an auxin gradient in a tissue, PILS are localized on the endoplasmic reticulum (ER) and might be involved in the regulation of intracellular auxin distribution and the cellular auxin level [23] [59] [60]. Compared to MdPIN1 which possesses a 300 amino acid-long central loop, the short loop (10 amino acids) of MdAECFP1 indicates that it may be a member of the PILS family. Both MdGH3s identified in the current study possess the functional domains of IAA-amido synthetase. The contrasting gene expression profiles between MdGH3.18 and MdGH3.102 indicated they functioned at different stages of fruit maturation in controlling the cellular level of free auxin, or the biologically active form of auxin, in apple fruit.

\subsection{Effect of Auxin Depends on Maturation Stage}

To understand the transcriptional changes of these investigated genes in response to exogenous IAA treatment, fruit from both cultivars was collected on the same date at 127 DAFB and then treated with IAA; and the gene expression patterns were characterized at 1, 3, 7, 14 days after IAA treatment. Due to the difference in the fruit maturation dynamics between these two cultivars, fruit of GD had a SPI of 2.3 and those of CP had a SPI of 1.0 when sampled. The expression of MdACS3 was not affected by exogenous IAA treatment in the fruit of GD. In contrast, it was significantly down-regulated in CP compared to that of non-treated fruit and with the maximum suppression at 7 days after IAA application. The expression of MdPIN1 was slightly up-regulated only in CP at 7 days after treatment, while MdAECFP1 was moderately up-regulated in GD until 14 days after treatment (Figure 3). Substantial suppression of MdGH3.102 expression was observed but only at 7 days after treatment
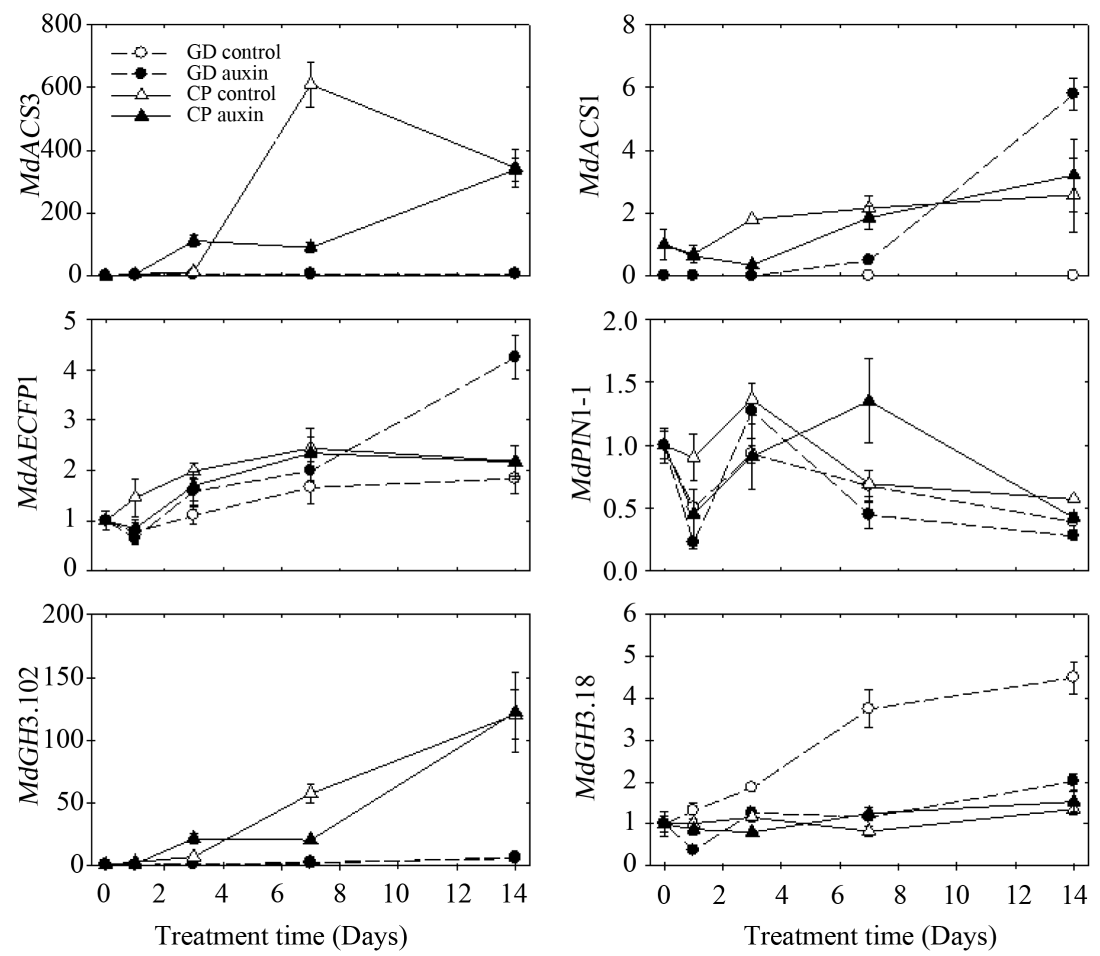

Figure 3. Gene expression profile of two ethylene biosynthesis genes (MdACS1 and $M d A C S 3)$ and four auxin efflux carrier and IAA-amido synthetase (MdPIN1, MdAECFP1, MdGH3.18, and MdGH3.102) in "Golden Delicious" (GD) and "Cripps Pink” (CP) apple fruit flesh (cortex) in response to exogenous IAA treatment. Numbers on $\mathrm{Y}$ axis indicate the fold change of the relative gene expression level; numbers on $\mathrm{X}$ axis indicate treatment time (days of incubation after IAA injection). The empty circle represents GD no IAA control; the black circle represents GD with $0.1 \mu \mathrm{M}$ IAA injection; the empty triangle represents $\mathrm{CP}$ no IAA control; the black triangle represents CP with $0.1 \mu \mathrm{M}$ IAA injection. Each data point represents the means of 4 replicates with standard error. 
in the fruit of CP. There was no observed effect on the expression of MdGH3.18 in CP, but its expression level was strongly down-regulated in GD. The different responses to exogenous IAA treatment on the specific gene expression patterns most likely reflect the maturation stage-dependent nature of inherent auxin metabolism between these two cultivars.

Although the mechanism behind the expression regulation due to exogenously applied auxin is not known, these observations were consistent with the assumption that the auxin per se inhibits ethylene biosynthesis, as indicated by the suppressed expression of the MdACS3 gene in CP; and conversely the reducing auxin level is required for activating the ethylene biosynthesis pathway in apple fruit. Exogenous IAA treatment appeared to have minor effect on the expression of both auxin efflux carrier genes, but a moderate suppression on IAAamido synthetase genes was observed (Figure 3). The less dramatic and delayed effects on the expression of most of these genes may be partly due to the fact that the auxin was applied into the core tissues of fruit, while the gene expression analysis was focused on the cortex tissues, where the most maturity data was measured. Interestingly, once the MdACS3 expression has already been induced such as in the fruit of GD, no suppression on the MdACS3 expression was observed due to IAA treatment. This observation also indirectly suggested that the link between MdACS3 and the reducing auxin level in apple cortex tissue as maturity progressed; and once its activated is irreversible. The expression of MdACS1 was induced 14 days after IAA treatment in the fruit of GD though it is not known if the activation of MdACS1 is due to the effect of expressed MdACS3 or directly from IAA treatment; a minimal effect on MdACS1 was observed in the fruit of CP. The stimulating effect of auxin on the ethylene biosynthesis pathway is consistent with the report [46] [47] on other fruit. A previous study also indicated that the detachment from the mother tree by harvesting fruit also stimulates the earlier activation of the MdACS1 gene, particularly for those fruits which are close to their physiological maturity [52]. Therefore, a complex relationship exists between auxin and ethylene functions, depending on gene family members and fruit developmental stages.

\section{Conclusion}

Based on the differential expression profiles of genes with annotated function of auxin transport and conjugation in maturing apple, the results provided the preliminary molecular evidences at transcriptional level on the potential connection between auxin availability and ethylene biosynthesis pathway activation. Cultivar-specific expression patterns of these genes in the fruit cortex tissues of two apple cultivars were shown to correlate with the fruit maturation process. In particular, the expression patterns of MdGH3.102 and MdAECFP1 coincided with the timing of ethylene biosynthesis pathway activation as indicated by the induction of MdACS3. As illustrated in Figure 4, our data suggested that the changing auxin level in apple fruit tissues may be one of the primary

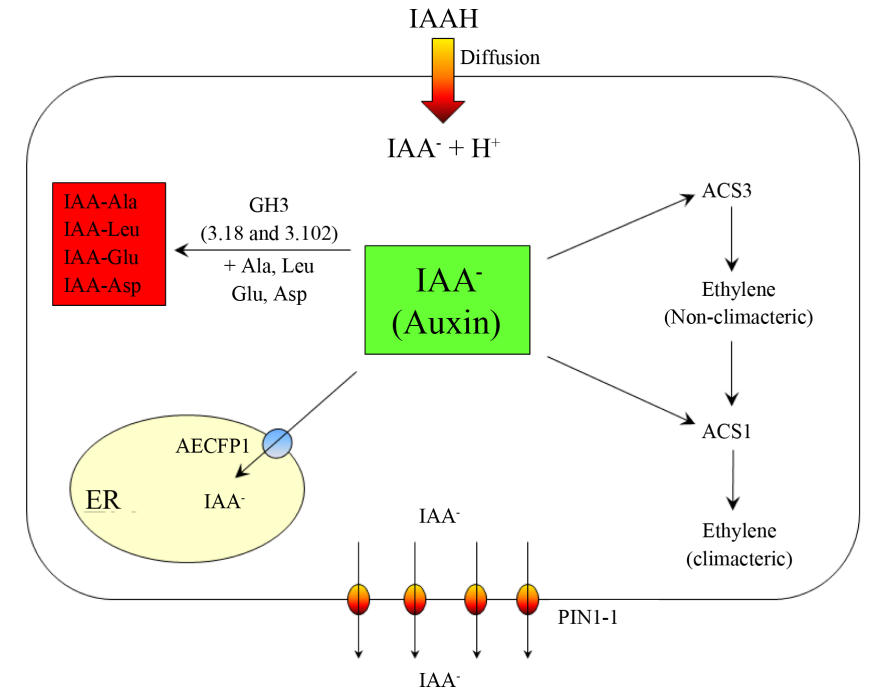

Figure 4. The proposed model of auxin and ethylene interaction in the cell of maturing apple fruit. Auxin efflux carriers (PIN1-1 and AECFP1) and IAA-amido synthetases (GH3) plays an essential role in regulating a cellular free auxin (IAA) level, which can affect the timing of activation of ethylene biosynthesis genes (ACS3 and ACS1). 
signals to trigger the expression of MdACS3 gene for pre-climacteric ethylene biosynthesis, and consequently the expression of MdACS1 for climacteric ripening. The likely scenario related auxin-ethylene relationship in maturing apple fruit is that while a certain level of auxin in apple fruit tissue (such as cortex) is needed for early fruit development (such as fruit cell division and expansion), the timely and efficient removal afterward is necessary for initiating fruit ripening process (such as the induction of MdACS3 gene for ethylene biosynthesis activation). Therefore, the control of auxin dynamics is a key regulation point of apple fruit ripening season. Although transcriptional regulation is the only initial step of gene function, the findings from this study are consistent with the hypothesis regarding the role of auxin availability and the potential auxin and ethylene interactions in regulating apple fruit ripening time. The allelic variations of these genes can differentiate the cultivar-specific dynamics of auxin in developing apple fruit, which in turn determines the maturation progression and the ripening season of an apple cultivar.

\section{Acknowledgements}

We thank Steve Reymond, Janie Countryman, Dave Buchanan and Edward Valdez for their excellent technical assistance. This work was supported by the funds from Washington Tree Fruit Research Committee.

\section{References}

[1] Giovannoni, J.J. (2004) Genetic Regulation of Fruit Development and Ripening. Plant Cell, 16, S170-S180. http://dx.doi.org/10.1105/tpc.019158

[2] McAtee, P., Karim, S., Schaffer, R. and David, K. (2013) A Dynamic Interplay between Phytohormones Is Required for Fruit Development, Maturation, and Ripening. Frontiers in Plant Science, 4, 79. http://dx.doi.org/10.3389/fpls.2013.00079

[3] Osorio, S., Scossa, F. and Fernie, A.R. (2013) Molecular Regulation of Fruit Ripening. Frontiers in Plant Science, 4, 198. http://dx.doi.org/10.3389/fpls.2013.00198

[4] Kazan, K. and Manners, J.M. (2008) Jasmonate Signaling: Toward an Integrated View. Plant Physiology, 146, 14591468. http://dx.doi.org/10.1104/pp.107.115717

[5] Kondo, S., Meemak, S., Ban, Y., Moriguchi, T. and Harada, T. (2009) Effects of Auxin and Jasmonates on 1-Aminocyclopropane-1-carboxylate (ACC) Synthase and ACC Oxidase Gene Expression during Ripening of Apple Fruit. Postharvest Biology and Technology, 51, 281-284. http://dx.doi.org/10.1016/j.postharvbio.2008.07.012

[6] Pauwels, L., Inze, D. and Goossens, A. (2009) Jasmonate-Inducible Gene: What Does It Mean? Trends in Plant Sciences, 14, 87-91. http://dx.doi.org/10.1016/j.tplants.2008.11.005

[7] Shinshi, H. (2008) Ethylene-Regulated Transcription and Crosstalk with Jasmonic Acid. Plant Science, 175, 18-23. http://dx.doi.org/10.1016/j.plantsci.2008.03.017

[8] Lau, O.W., Luk, S.F. and Wong, K.S. (1986) Background Correction Method for the Determination of Ascorbic Acid in Soft Drinks, Fruit Juices and Cordials Using Direct Ultraviolet Spectrophotometry. Analyst, 111, 665-670. http://dx.doi.org/10.1039/an9861100665

[9] Barry, C.S., Llop-Tous, M.I. and Grierson, D. (2000) The Regulation of 1-Aminocyclopropane-1-carboxylic Acid Synthase Gene Expression during the Transition from System-1 to System-2 Ethylene Synthesis in Tomato. Plant Physiology, 123, 979-986. http://dx.doi.org/10.1104/pp.123.3.979

[10] Oraguzie, N.C., Volz, R.K., Whitworth, C.J., Bassett, H.C.M., Hall, A.J. and Gardiner, S.E. (2007) Influence of MdACS1 Allelotype and Harvest Season within an Apple Germplasm Collection on Fruit Softening during Cold Air Storage. Postharvest Biology and Technology, 44, 212-219. http://dx.doi.org/10.1016/j.postharvbio.2006.12.013

[11] Rosenfield, C.L., Kiss, E. and Hrazdina, G. (1996) MdACS-2 (Accession No. U73815) and MdACS-3 (Accession No. U73816): Two New 1-Aminocyclopropane-1-carboxylate Synthase Sub Ripening Apple Fruit (PGR96-122). Plant Physiology, 112, 1735.

[12] Varanasi, V., Shin, S., Mattheis, J., Rudell, D. and Zhu, Y. (2011) Expression Profiles of the MdACS3 Gene Suggest a Function as an Accelerator of Apple (Malus $\times$ domestica) Fruit Ripening. Postharvest Biology and Technology, 62, 141-148. http://dx.doi.org/10.1016/j.postharvbio.2011.05.005

[13] Devoghalaere, F., Doucen, T., Guitton, B., Keeling, J., Payne, W., Ling, T.J., Ross, J.J., Hallett, I.C., Gunaseelan, K., Dayatilake, G.A., Diak, R., Breen, K.C., Tustin, D.S., Costes, E., Chagne, D., Schaffer, R.J. and David, K.M. (2012) A Genomics Approach to Understanding the Role of Auxin in Apple (Malus $\times$ domestica) Fruit Size Control. BMC Plant Biology, 12, 7. http://dx.doi.org/10.1186/1471-2229-12-7

[14] Zhu, Y., Zheng, P., Varanasi, V., Shin, S., Main, D., Curry, E. and Mattheis, J.P. (2012) Multiple Plant Hormones and 
Cell Wall Metabolism Regulate Apple Fruit Maturation Patterns and Texture Attributes. Tree Genetics \& Genomes, 8, 1389-1406. http://dx.doi.org/10.1007/s11295-012-0526-3

[15] Simon, S. and Petrasek, J. (2011) Why Plants Need More than One Type of Auxin. Plant Science, 180, 454-460. http://dx.doi.org/10.1016/j.plantsci.2010.12.007

[16] Zhao, Y. (2010) Auxin Biosynthesis and Its Role in Plant Development. Annual Review of Plant Biology, 61, 49-64. http://dx.doi.org/10.1146/annurev-arplant-042809-112308

[17] Ljung, K. (2013) Auxin Metabolism and Homeostasis during Plant Development. Development, 140, 943-950. http://dx.doi.org/10.1242/dev.086363

[18] Leyser, O. (2010) The Power of Auxin in Plants. Plant Physiology, 154, 501-505. http://dx.doi.org/10.1104/pp.110.161323

[19] Hayashi, K. (2012) The Interaction and Integration of Auxin Signaling Components. Plant and Cell Physiology, 53, 965-975. http://dx.doi.org/10.1093/pcp/pcs035

[20] Vernoux, T., Kronenberger, J., Grandjean, O., Laufs, P. and Traas, J. (2000) PIN-FORMED 1 Regulates Cell Fate at the Periphery of the Shoot Apical Meristem. Development, 127, 5157-5165.

[21] Xu, M., Zhu, L., Shou, H. and Wu, P. (2005) A PIN1 Family Gene, OsPIN1, Involved in Auxin-Dependent Adventitious Root Emergence and Tillering in Rice. Plant and Cell Physiology, 46, 1674-1681. http://dx.doi.org/10.1093/pcp/pci183

[22] Carraro, N., Forestan, C., Canova, S., Traas, J. and Varotto, S. (2006) ZmPIN1a and ZmPIN1b Encode Two Novel Putative Candidates for Polar Auxin Transport and Plant Architecture Determination of Maize. Plant Physiology, 142, 254-264. http://dx.doi.org/10.1104/pp.106.080119

[23] Zazimalova, E., Murphy, A.S., Yang, H., Hoyerova, K. and Hosek, P. (2010) Auxin Transporters-Why So Many? Cold Spring Harbor Perspectives in Biology, 2, a001552. http://dx.doi.org/10.1101/cshperspect.a001552

[24] Swarup, R. and Bennett, M. (2003) Auxin Transport: The Fountain of Life in Plants? Developmental Cell, 5, 824-826. http://dx.doi.org/10.1016/S1534-5807(03)00370-8

[25] Friml, J. (2010) Subcellular Trafficking of PIN Auxin Efflux Carriers in Auxin Transport. European Journal of Cell Biology, 89, 231-235. http://dx.doi.org/10.1016/j.ejcb.2009.11.003

[26] Feraru, E. and Friml, J. (2008) PIN Polar Targeting. Plant Physiology, 147, 1553-1559. http://dx.doi.org/10.1104/pp.108.121756

[27] Vanneste, S. and Friml, J. (2009) Auxin: A Trigger for Change in Plant Development. Cell, 136, 1005-1016. http://dx.doi.org/10.1016/j.cell.2009.03.001

[28] Korasick, D.A., Enders, T.A. and Strader, L.C. (2013) Auxin Biosynthesis and Storage Forms. Journal of Experimental Botany, 64, 2541-2555. http://dx.doi.org/10.1093/jxb/ert080

[29] Ding, X., Cao, Y., Huang, L., Zhao, J., Xu, C., Li, X. and Wang, S. (2008) Activation of the Indole-3-Acetic Acid-Amido Synthetase GH3-8 Suppresses Expansin Expression and Promotes Salicylate- and Jasmonate-Independent Basal Immunity in Rice. Plant Cell, 20, 228-240. http://dx.doi.org/10.1105/tpc.107.055657

[30] Seymour, G.B., Ostergaard, L., Chapman, N.H., Knapp, S. and Martin, C. (2013) Fruit Development and Ripening. Annual Review of Plant Biology, 64, 219-241. http://dx.doi.org/10.1146/annurev-arplant-050312-120057

[31] de Jong, M., Mariani, C. and Vriezen, W.H. (2009) The Role of Auxin and Gibberellin in Tomato Fruit Set. Journal of Experimental Botany, 60, 1523-1532. http://dx.doi.org/10.1093/jxb/erp094

[32] Srivastava, A. and Handa, A.K. (2005) Hormonal Regulation of Tomato Fruit Development: A Molecular Perspective. Journal of Plant Growth Regulation, 24, 67-82. http://dx.doi.org/10.1007/s00344-005-0015-0

[33] Stern, R.A., Flaishman, M., Applebaum, S. and Ben-Arie, R. (2007) Effect of Synthetic Auxins on Fruit Development of "Bing” Cherry (Prunus avium L.). Scientia Horticulturae, 114, 275-280. http://dx.doi.org/10.1016/j.scienta.2007.07.010

[34] Pattison, R.J. and Catala, C. (2012) Evaluating Auxin Distribution in Tomato (Solanum lycopersicum) through an Analysis of the PIN and AUX/LAX Gene Families. Plant Journal, 70, 585-598. http://dx.doi.org/10.1111/j.1365-313X.2011.04895.x

[35] Nishio, S., Moriguchi, R., Ikeda, H., Takahashi, H., Takahashi, H., Fujii, N., Guilfoyle, T.J., Kanahama, K. and Kanayama, Y. (2010) Expression Analysis of the Auxin Efflux Carrier Family in Tomato Fruit Development. Planta, 232, 755-764. http://dx.doi.org/10.1007/s00425-010-1211-0

[36] Mounet, F., Moing, A., Kowalczyk, M., Rohrmann, J., Petit, J., Garcia, V., Maucourt, M., Yano, K., Deborde, C., Aoki, K., Bergès, H., Granell, A., Fernie, A.R., Bellini, C., Rothan, C. and Lemaire-Chamley, M. (2012) Down-Regulation of a Single Auxin Efflux Transport Protein in Tomato Induces Precocious Fruit Development. Journal of Experimental Botany, 63, 4901-4917. http://dx.doi.org/10.1093/jxb/ers167 
[37] Dal Cin, V., Velasco, R. and Ramina, A. (2009) Dominance Induction of Fruitlet Shedding in Malus $\times$ domestica (L. Borkh): Molecular Changes Associated with Polar Auxin Transport. BMC Plant Biology, 9, 139. http://dx.doi.org/10.1186/1471-2229-9-139

[38] Schaffer, R.J., Ireland, H.S., Ross, J.J., Ling, T.J. and David, K.M. (2013) SEPALLATA1/2-Suppressed Mature Apples Have Low Ethylene, High Auxin and Reduced Transcription of Ripening-Related Genes. AoB Plants, 5, 1-10.

[39] Trainotti, L., Tadiello, A. and Casadoro, G. (2007) The Involvement of Auxin in the Ripening of Climacteric Fruits Comes of Age: The Hormone Plays a Role of Its Own and Has an Intense Interplay with Ethylene in Ripening Peaches. Journal of Experimental Botany, 58, 3299-3308. http://dx.doi.org/10.1093/jxb/erm178

[40] Zaharah, S., Singh, Z., Symons, G. and Reid, J. (2012) Role of Brassinosteroids, Ethylene, Abscisic Acid, and Indole3-Acetic Acid in Mango Fruit Ripening. Journal of Plant Growth Regulation, 31, 363-372. http://dx.doi.org/10.1007/s00344-011-9245-5

[41] Torrigiani, P., Bressanin, D., Beatriz Ruiz, K., Tadiello, A., Trainotti, L., Bonghi, C., Ziosi, V. and Costa, G. (2012) Spermidine Application to Young Developing Peach Fruits Leads to a Slowing Down of Ripening by Impairing Ripening-Related Ethylene and Auxin Metabolism and Signaling. Physiologia Plantarum, 146, 86-98. http://dx.doi.org/10.1111/j.1399-3054.2012.01612.x

[42] Symons, G.M., Chua, Y.J., Ross, J.J., Quittenden, L.J., Davies, N.W. and Reid, J.B. (2012) Hormonal Changes during Non-Climacteric Ripening in Strawberry. Journal of Experimental Botany, 63, 4741-4750. http://dx.doi.org/10.1093/jxb/ers147

[43] Pitts, R.J., Cernac, A. and Estelle, M. (1998) Auxin and Ethylene Promote Root Hair Elongation in Arabidopsis. Plant Journal, 16, 553-560. http://dx.doi.org/10.1046/j.1365-313x.1998.00321.X

[44] Rahman, A., Hosokawa, S., Oono, Y., Amakawa, T., Goto, N. and Tsurumi, S. (2002) Auxin and Ethylene Response Interactions during Arabidopsis Root Hair Development Dissected by Auxin Influx Modulators. Plant Physiology, 130, 1908-1917. http://dx.doi.org/10.1104/pp.010546

[45] Swarup, R., Parry, G., Graham, N., Allen, T. and Bennett, M. (2002) Auxin Cross-Talk: Integration of Signalling Pathways to Control Plant Development. Plant Molecular Biology, 49, 409-424. http://dx.doi.org/10.1023/A:1015250929138

[46] Ishiki, Y., Oda, A., Yaegashi, Y., Orihara, Y., Arai, T., Hirabayashi, T., Nakagawa, H. and Sato, T. (2000) Cloning of an Auxin-Responsive 1-Aminocyclopropane-1-carboxylate Synthase Gene (CMe-ACS2) from Melon and the Expression of ACS Genes in Etiolated Melon Seedlings and Melon Fruits. Plant Science, 159, 173-181. http://dx.doi.org/10.1016/S0168-9452(00)00298-3

[47] Tatsuki, M., Nakajima, N., Fujii, H., Shimada, T., Nakano, M., Hayashi, K.I., Hayama, H., Yoshioka, H. and Nakamura, Y. (2013) Increased Levels of IAA Are Required for System 2 Ethylene Synthesis Causing Fruit Softening in Peach (Prunus persica L. Batsch). Journal of Experimental Botany, 64, 1049-1059. http://dx.doi.org/10.1093/jxb/ers381

[48] Velasco, R., Zharkikh, A., Affourtit, J., Dhingra, A., Cestaro, A., Kalyanaraman, A., Fontana, P., Bhatnagar, S.K., Troggio, M., Pruss, D., Salvi, S., Pindo, M., Baldi, P., Castelletti, S., Cavaiuolo, M., Coppola, G., Costa, F., Cova, V., Dal Ri, A., Goremykin, V., Komjanc, M., Longhi, S., Magnago, P., Malacarne, G., Malnoy, M., Micheletti, D., Moretto, M., Perazzolli, M., Si-Ammour, A., Vezzulli, S., Zini, E., Eldredge, G., Fitzgerald, L.M., Gutin, N., Lanchbury, J., Macalma, T., Mitchell, J.T., Reid, J., Wardell, B., Kodira, C., Chen, Z., Desany, B., Niazi, F., Palmer, M., Koepke, T., Jiwan, D., Schaeffer, S., Krishnan, V., Wu, C., Chu, V.T., King, S.T., Vick, J., Tao, Q., Mraz, A., Stormo, A., Stormo, K., Bogden, R., Ederle, D., Stella, A., Vecchietti, A., Kater, M.M., Masiero, S., Lasserre, P., Lespinasse, Y., Allan, A.C., Bus, V., Chagne, D., Crowhurst, R.N., Gleave, A.P., Lavezzo, E., Fawcett, J.A., Proost, S., Rouze, P., Sterck, L., Toppo, S., Lazzari, B., Hellens, R.P., Durel, C.E., Gutin, A., Bumgarner, R.E., Gardiner, S.E., Skolnick, M., Egholm, M., Van de Peer, Y., Salamini, F. and Viola, R. (2010) The Genome of the Domesticated Apple (Malus $\times$ domestica Borkh.). Nature Genetics, 42, 833-839. http://dx.doi.org/10.1038/ng.654

[49] Brookfield, P., Murphy, P., Harker, R. and MacRae, E. (1997) Starch Degradation and Starch Pattern Indices; Interpretation and Relationship to Maturity. Postharvest Biology and Technology, 11, 23-30. http://dx.doi.org/10.1016/S0925-5214(97)01416-6

[50] Fan, X., Argenta, L. and Mattheis, J.P. (2002) Interactive Effects of 1-MCP and Temperature on "Elberta” Peach Quality. HortScience, 37, 134-138.

[51] Gasic, K., Hernandez, A. and Korban, S. (2004) RNA Extraction from Different Apple Tissues Rich in Polyphenols and Polysaccharides for cDNA Library Construction. Plant Molecular Biology Reporter, 22, 437-438. http://dx.doi.org/10.1007/BF02772687

[52] Zhu, Y., Rudell, D.R. and Mattheis, J.P. (2008) Characterization of Cultivar Differences in Alcohol Acyltransferase and 1-Aminocyclopropane-1-carboxylate Synthase Gene Expression and Volatile Ester Emission during Apple Fruit Maturation and Ripening. Postharvest Biology and Technology, 49, 330-339. http://dx.doi.org/10.1016/j.postharvbio.2008.03.015 
[53] Janick, J., Cummins, J.N., Brown, S.K. and Hemmat, M. (1996) Chapter 1: Apples. In: Janick, J. and Moore, J.N., Eds., Fruit Breed, Volume 1: Tree and Tropical Fruits, John Wiley \& Sons, Inc., Hoboken, 1-77.

[54] Varanasi, V., Shin, S., Johnson, F., Mattheis, J. and Zhu, Y. (2013) Differential Suppression of Ethylene Biosynthesis and Receptor Genes in “Golden Delicious” Apple by Preharvest and Postharvest 1-MCP Treatments. Journal of Plant Growth Regulation, 32, 585-595. http://dx.doi.org/10.1007/s00344-013-9326-8

[55] Lv, J., Rao, J., Johnson, F., Shin, S. and Zhu, Y. (2014) Genome-Wide Identification of Jasmonate Biosynthetic Genes and Characterization of Their Expression Profiles during Apple (Malus $\times$ domestica) Fruit Maturation. Plant Growth Regulation, 75, 355-364.

[56] Shin, S., Lv, J., Fazio, G., Mazzola, M. and Zhu, Y. (2014) Transcriptional Regulation of Ethylene and Jasmonate Mediated Defense Response in Apple (Malus domestica) Root during Pythium ultimum Infection. Horticulture Research, 1, 1-10.

[57] Frenkel, C. and Dyck, R. (1973) Auxin Inhibition of Ripening in Bartlett Pears. Plant Physiology, 51, 6-9. http://dx.doi.org/10.1104/pp.51.1.6

[58] Mousdale, D.M.A. and Knee, M. (1981) Indolyl-3-Acetic Acid and Ethylene Levels in Ripening Apple Fruits. Journal of Experimental Botany, 32, 753-758. http://dx.doi.org/10.1093/jxb/32.4.753

[59] Mravec, J., Skupa, P., Bailly, A., Hoyerova, K., Krecek, P., Bielach, A., Petrasek, J., Zhang, J., Gaykova, V., Stierhof, Y.D., Dobrev, P.I., Schwarzerova, K., Rolcik, J., Seifertova, D., Luschnig, C., Benkova, E., Zazimalova, E., Geisler, M. and Friml, J. (2009) Subcellular Homeostasis of Phytohormone Auxin Is Mediated by the ER-Localized PIN5 Transporter. Nature, 459, 1136-1140. http://dx.doi.org/10.1038/nature08066

[60] Barbez, E., Kubes, M., Rolcik, J., Beziat, C., Pencik, A., Wang, B., Rosquete, M.R., Zhu, J., Dobrev, P.I., Lee, Y., Zazimalova, E., Petrasek, J., Geisler, M., Friml, J. and Kleine-Vehn, J. (2012) A Novel Putative Auxin Carrier Family Regulates Intracellular Auxin Homeostasis in Plants. Nature, 485, 119-122. http://dx.doi.org/10.1038/nature11001 